$\mathrm{KEK} / \mathrm{TH} / 773$

June 2001

\title{
Non-commutative Gauge Theory, Open Wilson Lines and Closed Strings
}

\author{
Avinash Dhar ${ }^{1 *}$ and Yoshihisa Kitazawa ${ }^{\dagger}$ \\ Laboratory for Particle and Nuclear Physics, \\ High Energy Accelerator Research Organization (KEK), \\ Tsukuba, Ibaraki 305-0801, JAPAN.
}

\begin{abstract}
A recently proposed connection between closed string field and an open Wilson line defined on an arbitrary contour is further explored here. We suggest that reparametrization invariance of a Wilson line is the principle which determines the coupling of non-commutative gauge theory/matrix model to the modes of the closed string. An analogue of the level matching condition on the gauge theory/matrix model operators emerges quite naturally from the cyclic symmetry of the straight Wilson line. We show that the generating functional of correlation functions of these operators has the space-time gauge symmetry that one expects to find in closed string field theory. We also identify an infinite number of conserved operators in gauge theory/matrix model, the first of which is known to be the conserved stress tensor.
\end{abstract}

${ }^{1}$ On leave from Dept of Theoretical Phys, Tata Institute, Mumbai 400005, INDIA.

*adhar@post.kek.jp

† kitazawa@post.kek.jp 


\section{Introduction}

The subject of the coupling of non-commutative branes to closed strings in the bulk has been of much recent activity [1, 2, 3, 4, 5, 6] 1. At low energies, the fluctuations of non-commutative branes are controlled by a non-commutative gauge theory. So at low energies, the question of coupling of non-commutative branes to closed strings is equivalent to identifying gauge-invariant operators in the non-commutative gauge theory which couple to the different modes of the closed string. Gauge-invariant operators which couple to the tachyon and the massless modes of the closed string are now known and these consist of local operators smeared along straight Wilson lines [7]. Higher bulk modes are expected to follow a similar pattern.

It was pointed out in [8] that the non-commutative gauge theory operators dual to the tachyon and the massless modes in the case of the bosonic string could be obtained as the first two terms in a harmonic expansion of a Wilson line, based on a generic contour, around a certain straight line contour. As was suggested in [8], it seems natural to guess that the higher terms in the expansion give rise to gauge theory operators that couple to higher modes of the closed bosonic string. One may thus think of a Wilson line based on an arbitrary contour as the gauge theory operator dual to a bulk closed string. The purpose of the present work is to give concrete evidence for this proposal. Specifically what we show is that the operators that appear in the harmonic expansion of a Wilson line satisfy a kind of level matching condition and are in one-to-one correspondence with the modes of the closed bosonic string. Moreover, the corresponding source terms in the action have the linearized gauge symmetries expected in closed bosonic string field theory. Explicit disc amplitude calculations in an appropriate zero slope limit 9 confirm the general structure of the operators.

The plan of this paper is as follows. In the next section we discuss the above mentioned harmonic expansion of an arbitrary Wilson line. We suggest that the gauge theory operators that appear in the expansion couple to the different modes of closed string. We show that the cyclic symmetry of a straight Wilson line ensures a condition on these operators which is similar to the level matching condition for closed string modes. Thus, the gauge-theory operators we get in this way are in one-to-one correpondence with the modes of a closed string. In section 3 we discuss reparametrizations of the arbitrary contour underlying the Wilson line. This leads to reparametrizations of the straight line contour on which the operators involved in the expansion of the Wilson line are based, and hence these operators change under reparametrizations. However, we show that the correlation functions of these operators remain unchanged because of momentum conservation. In section 4 we discuss these operators from the point of view of the matrix model underlying non-commutative gauge theory. The structure of these operators is

\footnotetext{
${ }^{1}$ Other aspects of open Wilson lines were studied in [9, 10, 11, 12, 13, 14, 20].

${ }^{2}$ In this limit, described in section 5 , one gets leading terms in the gauge-invariant operators that couple to the corresponding modes of the closed string. It is these leading terms that are reproduced in the harmonic expansion of a Wilson line based on an arbitrary curve.
} 
much simpler in the matrix model setting and we are able to discuss some general features. In particular, we identify an infinite number of conserved operators, the first of which is known to be stress tensor of the matrix model. In section 5 we calculate some disc amplitudes to provide further evidence that the operators that appear in the harmonic expansion of an arbitrary Wilson line couple to the modes of the closed string. In section 6 we discuss the symmetries of the generating functional of the correlation functions of these operators. We show that reparametrization invariance of the original Wilson line ensures that this generating functional has the space-time gauge symmetries which one expects to find in closed string field theory. We conclude in section 7 with a discussion of our results and some open questions.

\section{Open Wilson lines and closed string modes}

In ordinary gauge theories, a generic gauge-invariant observable is provided by an arbitrary closed Wilson loop. Non-commutative gauge theories have more general gaugeinvariant observables, defined on open contours. Roughly speaking, these gauge-invariant observables can be written as Fourier transforms of open Wilson lines. In the operator formalism a generic open Wilson line is given by the following expression

$$
W_{C}[y]=\operatorname{Tr}\left(\mathrm{P} \exp \left\{i \int_{C} d \sigma \partial_{\sigma} y_{\mu}(\sigma) A_{\mu}(\hat{x}+y(\sigma))\right\} e^{i k . \hat{x}}\right)
$$

where

$$
\left[\hat{x}_{\mu}, \hat{x}_{\nu}\right]=i \theta_{\mu \nu}
$$

and the trace in (2.1) is over both the gauge group and the operator Hilbert space f The open Wilson line given by the above expression is gauge-invariant provided the momentum $k^{\mu}$ associated with it is fixed in terms of the straight line joining the end points of the path $C$, given by $y^{\mu}(\sigma)$ where $0 \leq \sigma \leq 1$, by the relation

$$
y_{\mu}(1)-y_{\mu}(0)=\theta_{\mu \nu} k_{\nu}
$$

The contour $C$ is otherwise completely arbitrary. This condition may be regarded as a boundary condition on the contours involved. A generic contour with this boundary condition may be parametrized as $y(\sigma)=\sigma(\theta k)+y^{\prime}(\sigma)$, where $0 \leq \sigma \leq 1$ and $y^{\prime}(\sigma)$ satisfies periodic boundary conditions. Thus the freedom contained in a generic Wilson line is exactly what is needed to describe a closed string!

In fact, this line of reasoning can be taken further. Let us confine our attention to smooth contours, with the additional condition that the tangents to the contour at the

\footnotetext{
${ }^{3}$ We will use the operator formulation throughout this paper. We are working with the fully noncommutative Euclidean case. See [8 for details of our notation and conventions.
} 
two ends are equal. In this case we may parametrize the contours as

$$
\begin{aligned}
y(\sigma) & =y_{0}(\sigma)+\theta w(\sigma) \\
y_{0}(\sigma) & =y_{0}(0)+\sigma l, l \equiv \theta k \\
w(\sigma) & =\sum_{n=1}^{\infty}\left(\alpha_{n} e^{-2 \pi i n \sigma}+\alpha_{n}^{*} e^{2 \pi i n \sigma}\right) .
\end{aligned}
$$

In the above parametrization the factor of $\theta$ in front of the periodic function $w_{\mu}(\sigma)$ has been chosen for later convenience. Also, we choose the constant $y_{0}(0)$ such that $w(\sigma)$ contains no zero mode.

Let us now expand the Wilson line, based on the given contour, around the Wilson line based on the straight line contour $C_{0}$ given by $y_{0}(\sigma)$. This gives

$$
\begin{aligned}
W_{C}[y]= & W_{C_{0}}\left[y_{0}\right]+\int_{0}^{1} d \sigma_{1}\left(\theta w\left(\sigma_{1}\right)\right)_{\mu}\left(\frac{\delta W_{C}[y]}{\delta y_{\mu}\left(\sigma_{1}\right)}\right)_{y=y_{0}} \\
& +\frac{1}{2 !} \int_{0}^{1} d \sigma_{1} \int_{0}^{1} d \sigma_{2}\left(\theta w\left(\sigma_{1}\right)\right)_{\mu}\left(\theta w\left(\sigma_{2}\right)\right)_{\nu}\left(\frac{\delta^{2} W_{C}[y]}{\delta y_{\mu}\left(\sigma_{1}\right) \delta y_{\nu}\left(\sigma_{2}\right)}\right)_{y=y_{0}}+\cdots
\end{aligned}
$$

The first term in the above equation is known to be the non-commutative gauge theory operator dual to the bulk closed string tachyon. The second term vanishes, since $\left(\frac{\delta W_{C}[y]}{\delta y_{\mu}(\sigma)}\right)_{y=y_{0}}$ is independent of $\sigma$, which can be easily verified using the cyclic symmetry of a straight Wilson line, and since $w(\sigma)$ has no zero mode. The first non-trivial contribution comes from the third term. Using the identity

$$
\begin{aligned}
\frac{\delta^{2} W_{C}[y]}{\delta y_{\mu}\left(\sigma_{1}\right) \delta y_{\nu}\left(\sigma_{2}\right)}=\operatorname{Tr}[ & \left(\hat{\mathcal{U}}_{C}\left(0, \sigma_{1}\right)\left(i \partial_{\sigma_{1}} y_{\lambda}\left(\sigma_{1}\right) \hat{F}_{\mu \lambda}\left(\hat{x}+y\left(\sigma_{1}\right)\right)\right) \hat{\mathcal{U}}_{C}\left(\sigma_{1}, \sigma_{2}\right)\right. \\
& \times\left(i \partial_{\sigma_{2}} y_{\rho}\left(\sigma_{2}\right) \hat{F}_{\nu \rho}\left(\hat{x}+y\left(\sigma_{2}\right)\right)\right) \hat{\mathcal{U}}_{C}\left(\sigma_{2}, 1\right) e^{i k . \hat{x}} \theta\left(\sigma_{2}-\sigma_{1}\right) \\
& \left.+\left(\sigma_{1} \leftrightarrow \sigma_{2}, \mu \leftrightarrow \nu\right)\right) \\
& +\hat{\mathcal{U}}_{C}\left(0, \sigma_{1}\right)\left(i \partial_{\sigma_{1}} y_{\lambda}\left(\sigma_{1}\right) \delta\left(\sigma_{1}-\sigma_{2}\right) \hat{D}_{\nu} \hat{F}_{\mu \lambda}\left(\hat{x}+y\left(\sigma_{1}\right)\right)\right) \hat{\mathcal{U}}_{C}\left(\sigma_{1}, 1\right) e^{i k . \hat{x}} \\
& \left.+\hat{\mathcal{U}}_{C}\left(0, \sigma_{1}\right)\left(i \partial_{\sigma_{1}} \delta\left(\sigma_{1}-\sigma_{2}\right) \hat{F}_{\mu \nu}\left(\hat{x}+y\left(\sigma_{1}\right)\right)\right) \hat{\mathcal{U}}_{C}\left(\sigma_{1}, 1\right) e^{i k . \hat{x}}\right]
\end{aligned}
$$

we may rewrite this term as

$$
\begin{aligned}
\theta_{\mu \mu^{\prime}} \theta_{\nu \nu^{\prime}}\left[\int_{0}^{1} d\right. & \sigma_{1} \int_{0}^{1} d \sigma_{2} w_{\mu}\left(\sigma_{1}\right) w_{\nu}\left(\sigma_{2}\right) \operatorname{Tr}\left(\hat{\mathcal{U}}_{C_{0}}\left(0, \sigma_{1}\right)\left(i l_{\lambda} \hat{F}_{\mu^{\prime} \lambda}\left(\hat{x}+y_{0}\left(\sigma_{1}\right)\right)\right) \hat{\mathcal{U}}_{C_{0}}\left(\sigma_{1}, \sigma_{2}\right)\right. \\
& \left.\times\left(i l_{\rho} \hat{F}_{\nu^{\prime} \rho}\left(\hat{x}+y_{0}\left(\sigma_{2}\right)\right)\right) \hat{\mathcal{U}}_{C_{0}}\left(\sigma_{2}, 1\right) e^{i k . \hat{x}} \theta\left(\sigma_{2}-\sigma_{1}\right)+\left(\sigma_{1} \leftrightarrow \sigma_{2}, \mu^{\prime} \leftrightarrow \nu^{\prime}\right)\right) \\
& +\int_{0}^{1} d \sigma_{1} w_{\mu}\left(\sigma_{1}\right) w_{\nu}\left(\sigma_{1}\right) \operatorname{Tr}\left(\hat{\mathcal{U}}_{C_{0}}\left(0, \sigma_{1}\right)\left(i l_{\lambda} \hat{D}_{\nu^{\prime}} \hat{F}_{\mu^{\prime} \lambda}\left(\hat{x}+y_{0}\left(\sigma_{1}\right)\right)\right) \hat{\mathcal{U}}_{C_{0}}\left(\sigma_{1}, 1\right) e^{i k . \hat{x}}\right) \\
& \left.+\int_{0}^{1} d \sigma_{1} w_{\mu}\left(\sigma_{1}\right) i \partial_{\sigma_{1}} w_{\nu}\left(\sigma_{1}\right) \operatorname{Tr}\left(\hat{\mathcal{U}}_{C_{0}}\left(0, \sigma_{1}\right) \hat{F}_{\mu^{\prime} \nu^{\prime}}\left(\hat{x}+y_{0}\left(\sigma_{1}\right)\right) \hat{\mathcal{U}}_{C_{0}}\left(\sigma_{1}, 1\right) e^{i k . \hat{x}}\right)\right]
\end{aligned}
$$


where $\hat{\mathcal{U}}_{C}\left(\sigma_{1}, \sigma_{2}\right)$ is the path-ordered phase factor, running along the contour $C$, from the point $\sigma_{1}$ to $\sigma_{2}$ t.

One crucial ingredient that we will now use is the cyclic symmetry of the Wilson line with the contour a straight line. It can be seen from this symmetry that the operator appearing inside the parametric integration in the first term in (2.7) depends only on the difference $\left(\sigma_{1}-\sigma_{2}\right)$ while the operators appearing in the other two terms are independent of $\sigma_{1}$. This ensures that when we substitute for $w(\sigma)$ from (2.4) in it we get an expression of the form

$$
\sum_{n=1}^{\infty} \alpha_{n \mu} \alpha_{n \nu}^{*} O_{\mu \nu}^{(n)}(k)
$$

which involves the modes $\alpha$ and $\alpha^{*}$ at the same mode number. Here $O_{\mu \nu}^{(n)}(k)$ are gauge theory operators which can be easily worked out from the expressions given above. For example, for $n=1$ the operator symmetric in the indices $\mu, \nu$ turns out to be precisely the operator that has been identified in [5] as dual to the bulk graviton in the bosonic string. The last term in (2.7) is purely antisymmetric in the indices $\mu, \nu$ and hence at the $n=1$ level it contributes only to the operator dual to the bulk antisymmetric tensor field.

The precise form of the gauge theory operators dual to the higher modes of the bosonic string has not been worked out, but the general form is expected to be similar to that for the tachyon and the massless modes. It is tempting to propose that these also appear in the harmonic expansion (2.5). Analogous to (2.8), a generic term in the expansion (2.5) has the form

$$
\sum_{n_{1}, n_{2}, \cdots=1}^{\infty} \sum_{m_{1}, m_{2}, \cdots=1}^{\infty} \alpha_{n_{1} \mu_{1}} \alpha_{n_{2} \mu_{2}} \cdots \alpha_{m_{1} \nu_{1}}^{*} \alpha_{m_{2} \nu_{2}}^{*} \cdots O_{\mu_{1}, \mu_{2}, \ldots ; \nu_{1}, \nu_{2}, \ldots}^{n_{1}, n_{2}, \ldots ; m_{1}, m_{2}, \ldots}(k) .
$$

The sum over the mode numbers in the above expression is not free but is constrained to satisfy $\sum_{j} n_{j}=\sum_{j} m_{j}$. As in the above example of $n=1$, this condition follows from the cylic symmetry of the straight Wilson line and is very similar to the level matching condition that the physical states of closed string satisfy, $\alpha_{n}$ and $\alpha_{n}^{*}$ being analogues of the two chiral modes of the closed string. Thus the gauge theory operators that appear in the harmonic expansion (2.5) are in one-to-one correspondence with the modes of closed bosonic string. Our proposal has, therefore, passed a crucial consistency check.

\section{Straight Wilson lines and reparametrization invariance}

A Wilson line operator based on any contour, by its definition, must not depend on any particular parametrization used for the contour. Since this is also true of the Wilson line

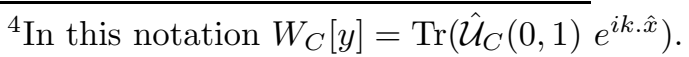


based on the generic contour, $W_{C}[y]$, this means that the sum on the right hand side of (2.5) should be independent of the parametrization used for the contour. The individual terms in the sum, however, are not required to satisfy this condition and may change under reparametrizations. Thus, in general the gauge theory operators appearing in (2.5) transform under reparametrizations. This would have been a problem for our proposal, but it turns out that the correlation functions of the operators remain unchanged, even though the operators themselves change. Let us see this in some detail.

Let us consider a different parametrization of the contour $C$ given by $y^{\prime}\left(\sigma^{\prime}\right), 0 \leq \sigma^{\prime} \leq 1$, with the boundary condition as before $y^{\prime}(1)-y^{\prime}(0)=l$. The harmonic expansion is now to be done around the corresponding straight line contour. The analogue of (2.4) is

$$
\begin{aligned}
y^{\prime}\left(\sigma^{\prime}\right) & =y_{0}^{\prime}\left(\sigma^{\prime}\right)+\theta w^{\prime}\left(\sigma^{\prime}\right) \\
y_{0}^{\prime}\left(\sigma^{\prime}\right) & =y_{0}^{\prime}(0)+\sigma^{\prime} l \\
w^{\prime}\left(\sigma^{\prime}\right) & =\sum_{n=1}^{\infty}\left(\alpha_{n}^{\prime} e^{-2 \pi i n \sigma^{\prime}}+\alpha_{n}^{\prime *} e^{2 \pi i n \sigma^{\prime}}\right)
\end{aligned}
$$

where $\alpha_{n}^{\prime}$ are the modes of $w^{\prime}\left(\sigma^{\prime}\right)$ which, as before, has no zero mode. For an infinitesimal reparametrization $\sigma^{\prime}=\sigma+\epsilon(\sigma)$, to the lowest order in $\epsilon$, using $y^{\prime}\left(\sigma^{\prime}\right)=y(\sigma)$ we get

$$
y_{0}^{\prime}(0)=y_{0}(0)-\epsilon_{0} l-2 \pi i \theta \sum_{n=1}^{\infty} n\left(\epsilon_{n} \alpha_{n}^{*}-\epsilon_{n}^{*} \alpha_{n}\right)
$$

and

$$
w^{\prime}(\sigma)=w(\sigma)-\epsilon(\sigma)\left(k+\partial_{\sigma} w(\sigma)\right) .
$$

The last equation holds only for non-zero modes with respect to $\sigma$, the zero mode of the product appearing in the second term on the right hand side being excluded from its consideration. Also, we have used the mode expansion $\epsilon(\sigma)=\epsilon_{0}+\sum_{n=1}^{\infty}\left(\epsilon_{n} e^{-2 \pi i n \sigma}+\right.$ $\left.\epsilon_{n}^{*} e^{2 \pi i n \sigma}\right)$ 円.

The harmonic expansion of the original Wilson line around the new straight line contour, $y_{0}^{\prime}\left(\sigma^{\prime}\right)$, is similar to $(2.5)$,

$$
\begin{aligned}
W_{C}\left[y^{\prime}\right] & =W_{C_{0}^{\prime}}\left[y_{0}^{\prime}\right]+\int_{0}^{1} d \sigma_{1}^{\prime}\left(\theta w^{\prime}\left(\sigma_{1}^{\prime}\right)\right)_{\mu}\left(\frac{\delta W_{C}\left[y^{\prime}\right]}{\delta y_{\mu}^{\prime}\left(\sigma_{1}^{\prime}\right)}\right)_{y^{\prime}=y_{0}^{\prime}} \\
& +\frac{1}{2 !} \int_{0}^{1} d \sigma_{1}^{\prime} \int_{0}^{1} d \sigma_{2}^{\prime}\left(\theta w^{\prime}\left(\sigma_{1}^{\prime}\right)\right)_{\mu}\left(\theta w^{\prime}\left(\sigma_{2}^{\prime}\right)\right)_{\nu}\left(\frac{\delta^{2} W_{C}\left[y^{\prime}\right]}{\delta y_{\mu}^{\prime}\left(\sigma_{1}^{\prime}\right) \delta y_{\nu}^{\prime}\left(\sigma_{2}^{\prime}\right)}\right)_{y^{\prime}=y_{0}^{\prime}}+\cdots .
\end{aligned}
$$

Since $\sigma_{1}^{\prime}, \sigma_{2}^{\prime}$, etc. are integration variables, we can drop the primes on them. Because of this the gauge theory operators involved in the above expansion differ from those involved in (2.5) only in that the starting point of the present straight line is shifted with respect

\footnotetext{
${ }^{5}$ Note that for $\sigma^{\prime}$ to range from 0 to 1 as $\sigma$ changes over the same range, we need $\epsilon(0)=\epsilon(1)=0$. This condition may be used to express the zero mode $\epsilon_{0}$ in terms of the other modes of $\epsilon(\sigma)$. Thus, $\epsilon_{0}$ is not an independent parameter.
} 
to the previous one by the amount given in (3.2). This difference can be removed by the shift $\hat{x} \rightarrow\left(\hat{x}+\epsilon_{0} l+2 \pi i \sum_{n=1}^{\infty} n\left(\epsilon_{n} \alpha_{n}^{*}-\epsilon_{n}^{*} \alpha_{n}\right)\right)$ at the expense of gaining the universal momentum-dependent phase

$$
e^{i l \cdot \sum_{n=1}^{\infty} 2 \pi i n\left(\alpha_{n} \epsilon_{n}^{*}-\alpha_{n}^{*} \epsilon_{n}\right)} .
$$

Thus, in terms of the modes $\alpha_{n}^{\prime}$ the above expansion looks like

$$
\sum_{n_{1}, n_{2}, \cdots=1}^{\infty} \sum_{m_{1}, m_{2}, \cdots=1}^{\infty} \alpha_{n_{1} \mu_{1}}^{\prime} \alpha_{n_{2} \mu_{2}}^{\prime} \cdots \alpha_{m_{1} \nu_{1}}^{\prime *} \alpha_{m_{2} \nu_{2}}^{\prime *} \cdots O_{\mu_{1}, \mu_{2}, \ldots ; \nu_{1}, \nu_{2}, \ldots}^{\prime n_{1}, n_{2}, \ldots ; m_{1}, m_{2}, \ldots}(k)
$$

where the new operators $O^{\prime}$ differ from the operators $O$ appearing in (2.9) only by the above phase.

One consequence of the above is that the correlation functions of the individual gauge theory operators, which enter the harmonic expansion of the Wilson line, are identical in any parametrization of the contour $C$. This is ensured by momentum conservation. It provides another consistency check for our proposal to identify these operators as dual to closed string modes. Since reparametrizations act in such a seemingly trivial fashion on the individual operators $O$, but since the modes $\alpha_{n}^{\prime}$ are non-trivially related to $\alpha_{n}$ by (3.3), one might ask how the sum in (3.6) manages to be equal to (2.9), as it must. To see how this happens it is instructive to study an example in detail, which is what we will do now.

Let us consider the term in (3.6) which correponds to the antisymmetric tensor field. The piece in this operator which is linear in field strength is

$$
\frac{i}{2 !} 2 \pi i \theta_{\mu \lambda} \theta_{\nu \rho}\left(\alpha_{1 \mu}^{\prime} \alpha_{1 \nu}^{\prime *}-\alpha_{1 \mu}^{\prime *} \alpha_{1 \nu}^{\prime}\right) \operatorname{Tr}\left(F_{\lambda \rho}\left(\hat{x}+y_{0}^{\prime}(0)\right) \hat{\mathcal{U}}_{C_{0}^{\prime}}(0,1) e^{i k . \hat{x}}\right)
$$

Using

$$
\alpha_{1}^{\prime}=\alpha_{1}-\epsilon_{1} k+2 \pi i \epsilon_{0} \alpha_{1}+2 \pi i \sum_{n=1}^{\infty}\left((n+1) \epsilon_{n}^{*} \alpha_{n+1}-n \epsilon_{n+1} \alpha_{n}^{*}\right),
$$

which can be obtained from (3.3), in (3.7), we find that it has the following extra terms over the corresponding term in (2.9)

$$
\begin{gathered}
2 \pi i \theta_{\rho \mu}\left(\alpha_{1 \mu} \epsilon_{1}^{*}-\alpha_{1 \mu}^{*} \epsilon_{1}\right) \operatorname{Tr}\left(i l_{\lambda} F_{\lambda \rho}\left(\hat{x}+y_{0}^{\prime}(0)\right) \hat{\mathcal{U}}_{C_{0}^{\prime}}(0,1) e^{i k . \hat{x}}\right) \\
\text { +terms quadratic in the modes. }
\end{gathered}
$$

Now, it is easy to see that

$$
\begin{aligned}
\operatorname{Tr}\left(i l_{\lambda} F_{\lambda \rho}\left(\hat{x}+y_{0}^{\prime}(0)\right) \hat{\mathcal{U}}_{C_{0}^{\prime}}(0,1) e^{i k \cdot \hat{x}}\right) & =-\frac{\delta W_{C_{0}^{\prime}}\left[y_{0}^{\prime}\right]}{\delta y_{0 \rho}^{\prime}(0)} \\
& =i k_{\rho} W_{C_{0}^{\prime}}\left[y_{0}^{\prime}\right]
\end{aligned}
$$


where in the first line above the variation with respect to $y_{0}^{\prime}(0)$ is done keeping $k$ fixed. Using this in (3.9) and retaining terms upto only first order in $\epsilon$, we get

$$
-i l .\left\{2 \pi i\left(\alpha_{1} \epsilon_{1}^{*}-\alpha_{1}^{*} \epsilon_{1}\right)\right\} W_{C_{0}}\left[y_{0}\right]+\text { terms quadratic in the modes. }
$$

Now, the tachyon term in (3.6) is $W_{C_{0}^{\prime}}\left[y_{0}^{\prime}\right]$ which, as discussed above, differs from $W_{C_{0}}\left[y_{0}\right]$ by the phase in (3.5). To lowest order in $\epsilon_{1}$ this gives an extra term which precisely cancels the extra contribution linear in modes we have found above in (3.11). This example illustrates how the extra phase and the change in the modes due to a reparametrization compensate each other. We expect this to happen for all the other terms as well since this is required by the reparametrization invariance of the Wilson line $W_{C}$. A general proof will be given in the next section where we discuss the connection with the underlying matrix model.

\section{Relation with matrix model}

It is useful to relate the above considerations to an underlying bosonic matrix model ?. The Wilson line of the non-commutative gauge theory discussed above may be related to the Wilson line of this matrix model,

$$
\tilde{W}_{C}[y]=\operatorname{Tr}\left(\mathrm{P} \exp \left\{-i \int d \sigma \partial_{\sigma} y_{\mu}(\sigma) \theta_{\mu \nu}^{-1} X_{\nu}\right\}\right)
$$

using the by now familiar procedure of expanding around the appropriate brane solution, $X_{\mu}=\hat{x}_{\mu}-\theta_{\mu \nu} A_{\nu}(\hat{x})$, where $\hat{x}_{\mu}$ satisfies the commutation relation given in (2.2). It turns out that $\tilde{W}_{C}$ is not exactly the same as $W_{C}$ of (2.1). However, the difference is only a phase which depends on the contour. That is \,

$$
\tilde{W}_{C}[y]=e^{i \phi_{C}[y]} W_{C}[y]
$$

where

$$
\phi_{C}[y]=\frac{1}{2} k \cdot y(0)+\frac{1}{2} \int_{0}^{1} d \sigma y(\sigma) \theta^{-1} \partial_{\sigma} y(\sigma)
$$

An interesting aspect of above the phase can be seen by using the parametrization (2.4) in it. This gives

$$
\phi_{C}[y]=k \cdot(y(0)-\theta w(0))-\frac{1}{2} \int_{0}^{1} d \sigma w(\sigma) \theta \partial_{\sigma} w(\sigma) .
$$

\footnotetext{
${ }^{6}$ The relevant matrix model is the IIB type [15. Possible existence of bosonic M theory has been speculated in 16, 17, 18, 19.

${ }^{7}$ Similar expressions have appeared earlier in [20, 21]. Such an expression is naturally obtained in the boundary state formalism.
} 
Using $y(0)=y_{0}(0)+\theta w(0)$ in this, substituiting in (4.2) and absorbing part of the the phase, $e^{i k \cdot y_{0}(0)}$, in the Wilson line by shifting to origin the starting point of the corresponding straight line contour, we get

$$
\tilde{W}_{C}[y]=e^{-\frac{i}{2} \int_{0}^{1} d \sigma w(\sigma) \theta \partial_{\sigma} w(\sigma)} W_{k}[w] .
$$

Here, we have used the notation $W_{k}[w]$ to indicate that the straight line path involved in the Wilson line is now given simply by $\sigma l$, the dependence on the starting point $y_{0}(0)$ having disappeared from it.

The operator $W_{k}[w]$ is not reparametrization invariant, though, of course, the entire right hand side of (4.5) does have this property. This is because the original Wilson line operator $W_{C}[y]$ and the phase $\phi_{C}[y]$ are independently reparametrization invariant ${ }^{8}$. Therefore, a physically meaningful harmonic expansion of the Wilson line that naturally appears in the matrix model involves expanding $W_{k}[w]$ together with the $w$-dependent phase in (4.5). This gives gauge theory operators that are different from the operators that appear in the expansion (2.9). One of the differences is that the $w$-dependent phase in (4.5) contributes additional terms to the various operators. For example, at $n=1$ the linear field strength piece in the operator that couples to the antisymmetric tensor is changed from $F_{\mu \nu}\left(\hat{x}+y_{0}(0)\right)$ to $\left(F_{\mu \nu}(\hat{x})+\theta_{\mu \nu}^{-1}\right)$, attached to the straight Wilson line. The other difference, which we have already mentioned above, is that the straight line contour on which the new operators are based is determined solely by the momentum $k$. This has the important consequence that the new operators are invariant under reparametrizations, since the only source of reparametrization dependence in the operators that appear in (2.9) is the starting point of the straight line contour, $y_{0}(0)$. Since the modes of the periodic function $w$ change under reparametrizations, one might wonder as to how the sum in (2.9) manages to be reparametrization invariant, which it must be since the Wilson line is by definition reparametrization invariant. Later on in this section we will see how this happens.

Instead of doing a harmonic expansion of the right hand side of (4.5), one could directly expand (4.1) itself. This gives matrix model expressions for the gauge theory operators involved in the harmonic expansion. These expressions are much simpler than the expressions that appear in, for example (2.7), and the general structure of the operators is more transparent. So it is useful to discuss the harmonic expansion of (4.1), which is what we shall do now.

One property of the matrix model expression for the Wilson line is that it depends only on the parametric derivative of the function $y(\sigma)$ that characterizes the contour. Because of this, its harmonic expansion is most easily obtained in terms of the derivative of the periodic function $w(\sigma)$. In fact, it is easy to see that

$\tilde{W}_{C}[y]=\operatorname{Tr}\left(e^{i k \cdot X}\right)+\int d \sigma i \partial_{\sigma} w_{\mu}(\sigma) \operatorname{Tr}\left(e^{i \sigma k \cdot X} X_{\mu} e^{i(1-\sigma) k \cdot X}\right)$

\footnotetext{
${ }^{8}$ Note that the phase $e^{-i k . y(0)}$ is reparametrization invariant.
} 


$$
\begin{aligned}
& +\int_{0}^{1} d \sigma_{1} \int_{\sigma_{1}}^{1} d \sigma_{2} i \partial_{\sigma_{1}} w_{\mu}\left(\sigma_{1}\right) i \partial_{\sigma_{2}} w_{\nu}\left(\sigma_{2}\right) \operatorname{Tr}\left(e^{i \sigma_{1} k \cdot X} X_{\mu} e^{i\left(\sigma_{2}-\sigma_{1}\right) k \cdot X} X_{\nu} e^{i\left(1-\sigma_{2}\right) k \cdot X}\right) \\
& +\cdots
\end{aligned}
$$

The first term in the above expansion is just the operator that couples to the tachyon. The second term vanishes because the operator involved is independent of $\sigma$ due to the cyclic property of the trace. Substituiting the mode expansion for $w(\sigma)$ in the third term gives the following:

$$
\sum_{n=1}^{\infty} \alpha_{n \mu}^{*} \alpha_{n \nu}(2 \pi n)^{2} \int_{0}^{1} d \sigma e^{-2 \pi i n \sigma} \operatorname{Tr}\left(X_{\mu} e^{i \sigma k \cdot X} X_{\nu} e^{i(1-\sigma) k \cdot X}\right)
$$

From this expansion we may now read off the matrix model operators that couple to specific modes of the closed string. As before, the analogue of level matching condition emerges because of the cyclic symmetry of the trace. As an example, the operators that couple to the massless modes are given by the $n=1$ term in (4.7). The part of this term symmetric in $\mu, \nu$ has been shown in [5] to give the stress tensor of the matrix model.

The general structure of the operators involved in the expansion (4.6) is easy to write down. The operator in the $(\mathrm{r}+1)$ th term is

$$
\begin{aligned}
\int_{0}^{1} d \sigma_{1} e^{\mp 2 \pi i n_{1} \sigma_{1}} \int_{\sigma_{1}}^{1} d \sigma_{2} e^{\mp 2 \pi i n_{2} \sigma_{2}} \cdots \int_{\sigma_{r-1}}^{1} d \sigma_{r} e^{\mp 2 \pi i n_{r} \sigma_{r}} \\
\times \operatorname{Tr}\left(e^{i \sigma_{1} k \cdot X} X_{\mu_{1}} e^{i\left(\sigma_{2}-\sigma_{1}\right) k \cdot X} X_{\mu_{2}} \cdots e^{i\left(1-\sigma_{r}\right) k \cdot X}\right)
\end{aligned}
$$

The $\mp$ signs in the exponents correspond to mode $\alpha$ or its conjugate $\alpha^{*}$. The above expression is non-vanishing only if the signs are chosen such that the sum $\sum_{l} n_{l}$ for the positive signs equals that for the negative signs ${ }^{9}$. This is the analogue of the level matching condition for the general term in the expansion (4.6).

It is easy to see that the operators that appear in the expansion of the Wilson line $\tilde{W}_{C}$ are invariant under reparametrizations, even though the modes of the periodic function that characterizes the contour $C$ transform in a complicated way. It is then natural to ask as to how the sum in (4.6) still manages to be reparametrization invariant, as it must, since $\tilde{W}_{C}$ is by definition reparametrization invariant. To see how this comes about, let us consider a non-trivial example in detail.

The change in the harmonic function $w(\sigma)$ under reparametrizations is given by the expression in (3.3). Consider now the first non-trivial term in the expansion (4.6), namely, the third term in this equation. It is easy to see that the change in this term due to the first term in the variation of $w$ vanishes by itself. Its variation due to the second term in the variation of $w$ is given by

$$
\int_{0}^{1} d \sigma_{1} \int_{\sigma_{1}}^{1} d \sigma_{2}\left\{\partial_{\sigma_{1}}\left(-i \epsilon\left(\sigma_{1}\right) \dot{w}_{\mu}\left(\sigma_{1}\right)\right) i \dot{w}_{\nu}\left(\sigma_{2}\right)+i \dot{w}_{\mu}\left(\sigma_{1}\right) \partial_{\sigma_{2}}\left(-i \epsilon\left(\sigma_{2}\right) \dot{w}_{\nu}\left(\sigma_{2}\right)\right)\right\}
$$

\footnotetext{
${ }^{9}$ In the operators that appear in the expansion this expression has to be appropriately symmetrized by permuting the indices and the mode exponentials. The statement here holds only for the symmetrized operators like, for example, in (5.6).

${ }^{10}$ Here, a dot represents a derivative with respect to the argument.
} 


$$
\times \operatorname{Tr}\left(e^{i \sigma_{1} k \cdot X} X_{\mu} e^{i\left(\sigma_{2}-\sigma_{1}\right) k \cdot X} X_{\nu} e^{i\left(1-\sigma_{2}\right) k \cdot X}\right)
$$

This is clearly non-vanishing and hence for consistency must be cancelled in the variation of the sum (4.6). It turns out that the variation of the next term in this sum under the first term in the variation of $w$ does not vanish. In fact, the result is a contribution that precisely cancels the above contribution, as we shall see now.

The next term in the sum (4.6) is

$$
\begin{array}{rl}
\int_{0}^{1} d \sigma_{1} \int_{\sigma_{1}}^{1} & d \sigma_{2} \int_{\sigma_{2}}^{1} d \sigma_{3} i \dot{w}_{\mu}\left(\sigma_{1}\right) i \dot{w}_{\nu}\left(\sigma_{2}\right) i \dot{w}_{\lambda}\left(\sigma_{3}\right) \\
& \times \operatorname{Tr}\left(e^{i \sigma_{1} k \cdot X} X_{\mu} e^{i\left(\sigma_{2}-\sigma_{1}\right) k \cdot X} X_{\nu} e^{i\left(\sigma_{3}-\sigma_{2}\right) k \cdot X} X_{\lambda} e^{i\left(1-\sigma_{3}\right) k \cdot X}\right)
\end{array}
$$

The change in this under the first term in the variation of $w$ is

$$
\begin{aligned}
& \int_{0}^{1} d \sigma_{1} \int_{\sigma_{1}}^{1} d \sigma_{2} \int_{\sigma_{2}}^{1} d \sigma_{3}\left\{\left(-i \dot{\epsilon}\left(\sigma_{1}\right) k_{\mu}\right) i \dot{w}_{\nu}\left(\sigma_{2}\right) i \dot{w}_{\lambda}\left(\sigma_{3}\right)+i \dot{w}_{\mu}\left(\sigma_{1}\right)\left(-i \dot{\epsilon}\left(\sigma_{2}\right) k_{\nu}\right) i \dot{w}_{\lambda}\left(\sigma_{3}\right)\right. \\
& \left.+i \dot{w}_{\mu}\left(\sigma_{1}\right) i \dot{w}_{\nu}\left(\sigma_{2}\right)\left(-i \dot{\epsilon}\left(\sigma_{3}\right) k_{\lambda}\right)\right\} \operatorname{Tr}\left(e^{i \sigma_{1} k . X} X_{\mu} e^{i\left(\sigma_{2}-\sigma_{1}\right) k \cdot X} X_{\nu} e^{i\left(\sigma_{3}-\sigma_{2}\right) k \cdot X} X_{\lambda} e^{i\left(1-\sigma_{3}\right) k . X}\right)
\end{aligned}
$$

Now, partially integrating the derivative on $\epsilon$ and using $\epsilon(0)=\epsilon(1)=0$, we find that the middle term in the curly brackets above gives two contributions, which combine with the contributions of the first and the last terms to give

$$
\begin{gathered}
\int_{0}^{1} d \sigma_{1} \int_{\sigma_{1}}^{1} d \sigma_{2}\left\{\left(-i \epsilon\left(\sigma_{1}\right) \dot{w}_{\mu}\left(\sigma_{1}\right)\right) i \dot{w}_{\nu}\left(\sigma_{2}\right) \partial_{\sigma_{1}} \operatorname{Tr}\left(e^{i \sigma_{1} k \cdot X} X_{\mu} e^{i\left(\sigma_{2}-\sigma_{1}\right) k \cdot X} X_{\nu} e^{i\left(1-\sigma_{2}\right) k \cdot X}\right)\right. \\
\left.+i \dot{w}_{\mu}\left(\sigma_{1}\right)\left(-i \epsilon\left(\sigma_{2}\right) \dot{w}_{\nu}\left(\sigma_{2}\right)\right) \partial_{\sigma_{2}} \operatorname{Tr}\left(e^{i \sigma_{1} k \cdot X} X_{\mu} e^{i\left(\sigma_{2}-\sigma_{1}\right) k \cdot X} X_{\nu} e^{i\left(1-\sigma_{2}\right) k \cdot X}\right)\right\}
\end{gathered}
$$

Partially integrating the derivatives and again using that $\epsilon(0)=\epsilon(1)=0$, we find that this precisely cancels the contribution in (4.9).

The above calculation illustrates the general mechanism by which the sum in (4.6) manages to be reparametrization invariant. The structure of the operators that appear in the sum is such that the contribution of the term at a given order under the second term in the variation of $w$ is cancelled by the contribution of the next higher order term due to the first term in the variation of $w$. In this respect the third term in (4.6) is rather special. For this term there is no lower order term whose contribution would cancel its contribution under the $-\epsilon k$ term in the variation of $w$. This contribution must, therefore, vanish by itself. This ensures the existence of an infinite number of operators, given by the expression

$$
O_{\mu \nu}^{(n)}(k)=\int_{0}^{1} d \sigma e^{-2 \pi i n \sigma} \operatorname{Tr}\left(X_{\mu} e^{i \sigma k \cdot X} X_{\nu} e^{i(1-\sigma) k \cdot X}\right), n=1,2, \cdots,
$$

which satisfy the conservation equation

$$
k_{\mu} O_{\mu \nu}^{(n)}(k)=0 .
$$


The proof of this conservation equation is trivial and completely kinematical.

The part of the operator for $n=1$ which is symmetric in the indices $\mu, \nu$ is just the stress tensor of the matrix model. As noted in [5], the proof of the conservation of the stress tensor does not require the equation of motion of the matrix model to be satisfied. This is because in the zero slope limit conformal invariance of disc amplitudes does not impose any restrictions on the gauge field. Here we have seen this conservation equation emerging as a direct consequence of the reparametrization invariance of the Wilson line. From the space-time point of view, the conservation of the stress tensor is equivalent to the decoupling of the longitudinal mode of the graviton. The conservation equation (4.14) for operators with $n \geq 2$ might at first seem surprising. However, it turns out that this is not only required by reparametrization invariance, as discussed above, but also because of some unexpected identities satisfied by higher tensor operators. This will be discussed in more detail at the end of the next section.

\section{$5 \quad$ Disc amplitudes and Wilson line operators}

In this section we would like to provide further evidence in support of our suggestion that the matrix model operators (4.8) couple to different modes of the closed string. This evidence comes from explicit calculations of disc amplitudes with the insertion of a single closed string vertex operator, in the appropriate zero slope limit. Since we are interested in the (bosonic) matrix model for D-instantons, we have no directions parallel to the brane, and so the calculations involve only the scalar fields and no gauge fields.

We wish to calculate disk amplitudes of the type

$$
<V(z) \operatorname{Tr}\left(\mathrm{P} \exp \left\{i \int_{-\infty}^{+\infty} d t \Phi_{\mu} i \partial_{\perp} x^{\mu}(t)\right\}\right)>
$$

Here $V(z)$ is a closed string vertex operator, the trace is over the matrix $\Phi_{\mu}$, representing directions perpendicular to the multiple D-instantons that we are considering, and $\partial_{\perp}$ is a derivative in the direction transverse to the boundary of the world-sheet. With Dirichlet boundary conditions on $x^{\mu}$, the propagator is given by

$$
<x^{\mu}(z) x^{\nu}(w)>=-\alpha^{\prime} g^{\mu \nu}\{\log |z-w|-\log |z-\bar{w}|\}
$$

The calculations now proceed similarly to those done in [5] for the bosonic string.

Consider first the closed string tachyon vertex operator $V(z)=e^{i k \cdot x(z)}$. Using (5.2) we get

$$
\begin{aligned}
& <e^{i k \cdot x(z)} \operatorname{Tr}\left(\mathrm{P} \exp \left(i \int_{-\infty}^{+\infty} d t \Phi_{\mu} i \partial_{\perp} x^{\mu}(t)\right)\right)> \\
& \sim \operatorname{Tr}\left(e^{2 \pi i \alpha^{\prime} k \cdot \Phi}\right) \\
& \equiv \operatorname{Tr}\left(e^{i k \cdot X}\right) .
\end{aligned}
$$


Here we have used that

$$
\begin{aligned}
i \partial_{\perp}\{\log |z-t|-\log |\bar{z}-t|\} & =\frac{(z-\bar{z})}{(z-t)(\bar{z}-t)} \\
& \equiv 2 \pi i \partial_{t} \tau(t, z)
\end{aligned}
$$

where, for a fixed value of $z$, the function $\frac{1}{2 \pi i} \log \left(\frac{t-z}{t-\bar{z}}\right) \equiv \tau(t, z)$ is monotonic in $t$ and satisfies $\tau(\infty, z)-\tau(-\infty, z)=1$. In the last step in (5.3) we have used the definition $X \equiv 2 \pi \alpha^{\prime} \Phi$ to match with the expressions in the previous section. Also, we have ignored other factors since we are only interested in that part of the result of the computation which involves the matrix model variables.

We next consider the vertex operators at the massless level of the closed string, $V^{\mu \nu}(z)=\partial x^{\mu} \bar{\partial} x^{\nu} e^{i k \cdot x(z)}$. Using

$$
\begin{gathered}
<\partial x^{\mu}(z) i \partial_{\perp} x^{\nu}(t)>=-\frac{\alpha^{\prime} g^{\mu \nu}}{(z-t)^{2}}=-\frac{2 \pi i \alpha^{\prime} g^{\mu \nu}}{(z-\bar{z})} e^{-2 \pi i \tau(t, z)} \partial_{t} \tau(t, z) \\
<\bar{\partial} x^{\mu}(\bar{z}) i \partial_{\perp} x^{\nu}(t)>=\frac{\alpha^{\prime} g^{\mu \nu}}{(\bar{z}-t)^{2}}=\frac{2 \pi i \alpha^{\prime} g^{\mu \nu}}{(z-\bar{z})} e^{2 \pi i \tau(t, z)} \partial_{t} \tau(t, z)
\end{gathered}
$$

we get

$$
\begin{aligned}
& <\partial x^{\mu} \bar{\partial} x^{\nu} e^{i k \cdot x(z)} \operatorname{Tr}\left(\mathrm{P} \exp \left\{i \int_{-\infty}^{+\infty} d t \Phi_{\mu} i \partial_{\perp} x^{\mu}(t)\right\}\right)> \\
\sim & \int_{0}^{1} d \tau_{1} e^{-2 \pi i \tau_{1}} \int_{\tau_{1}}^{1} d \tau_{2} e^{2 \pi i \tau_{2}} \operatorname{Tr}\left(e^{i \tau_{1} k \cdot X} X^{\mu} e^{i\left(\tau_{2}-\tau_{1}\right) k \cdot X} X^{\nu} e^{i\left(1-\tau_{2}\right) k \cdot X}\right) \\
& +\int_{0}^{1} d \tau_{1} e^{2 \pi i \tau_{1}} \int_{\tau_{1}}^{1} d \tau_{2} e^{-2 \pi i \tau_{2}} \operatorname{Tr}\left(e^{i \tau_{1} k \cdot X} X^{\nu} e^{i\left(\tau_{2}-\tau_{1}\right) k \cdot X} X^{\mu} e^{i\left(1-\tau_{2}\right) k \cdot X}\right) .
\end{aligned}
$$

At higher levels, there are several different types of closed string vertex operators. For example, at the next level there are the following three different types of vertex operators,

$$
\begin{aligned}
V_{1}^{\mu \nu \lambda \rho}(z) & =\partial x^{\mu} \partial x^{\nu} \bar{\partial} x^{\lambda} \bar{\partial} x^{\rho} e^{i k \cdot x(z)} \\
V_{2}^{\mu \nu \lambda}(z) & =\partial x^{\mu} \partial x^{\nu} \bar{\partial}^{2} x^{\lambda} e^{i k \cdot x(z)} \\
V_{3}^{\mu \nu}(z) & =\partial^{2} x^{\mu} \bar{\partial}^{2} x^{\nu} e^{i k \cdot x(z)} .
\end{aligned}
$$

Inserting the first of these in (5.1) and doing the computations as above, we get

$$
\begin{aligned}
<V_{1}^{\mu \nu \lambda \rho}(z) \operatorname{Tr}\left(\mathrm{P} \exp \left\{i \int_{-\infty}^{+\infty} d t \Phi_{\mu} i \partial_{\perp} x^{\mu}(t)\right\}\right)> \\
\sim \int_{0}^{1} d \tau_{1} e^{-2 \pi i \tau_{1}} \int_{\tau_{1}}^{1} d \tau_{2} e^{-2 \pi i \tau_{2}} \int_{\tau_{2}}^{1} d \tau_{3} e^{2 \pi i \tau_{3}} \int_{\tau_{3}}^{1} d \tau_{4} e^{2 \pi i \tau_{4}} \\
\times \operatorname{Tr}\left(e^{i \tau_{1} k \cdot X} X^{\mu} e^{i\left(\tau_{2}-\tau_{1}\right) k \cdot X} X^{\nu} e^{i\left(\tau_{3}-\tau_{2}\right) k \cdot X} X^{\lambda} e^{i\left(\tau_{4}-\tau_{3}\right) k \cdot X} X^{\rho} e^{i\left(1-\tau_{4}\right) k \cdot X}\right) \\
+\cdots
\end{aligned}
$$

The dots stand for other terms which are obtained, like the second term on the right hand side of (5.6), by permuting the indices on the $X$ 's together with the signs in the mode 
exponentials. Similarly, for the other two operators in (5.7), using (5.5) and the identity $1 /(t-z)=\left(e^{-2 \pi i \tau}-1\right) /(z-\bar{z})$, we get

$$
\begin{aligned}
& <V_{2}^{\mu \nu \lambda}(z) \operatorname{Tr}\left(\mathrm{P} \exp \left\{i \int_{-\infty}^{+\infty} d t \Phi_{\mu} i \partial_{\perp} x^{\mu}(t)\right\}\right)> \\
\sim & \int_{0}^{1} d \tau_{1} e^{-2 \pi i \tau_{1}} \int_{\tau_{1}}^{1} d \tau_{2} e^{-2 \pi i \tau_{2}} \int_{\tau_{2}}^{1} d \tau_{3} e^{4 \pi i \tau_{3}} \\
& \times \operatorname{Tr}\left(e^{i \tau_{1} k \cdot X} X^{\mu} e^{i\left(\tau_{2}-\tau_{1}\right) k \cdot X} X^{\nu} e^{i\left(\tau_{3}-\tau_{2}\right) k \cdot X} X^{\lambda} e^{i\left(1-\tau_{3}\right) k \cdot X}\right) \\
& +\cdots,
\end{aligned}
$$

and

$$
\begin{aligned}
& <V_{3}^{\mu \nu}(z) \operatorname{Tr}\left(\mathrm{P} \exp \left\{i \int_{-\infty}^{+\infty} d t \Phi_{\mu} i \partial_{\perp} x^{\mu}(t)\right\}\right)> \\
\sim & \int_{0}^{1} d \tau_{1} e^{-4 \pi i \tau_{1}} \int_{\tau_{1}}^{1} d \tau_{2} e^{4 \pi i \tau_{2}} \operatorname{Tr}\left(e^{i \tau_{1} k \cdot X} X^{\mu} e^{i\left(\tau_{2}-\tau_{1}\right) k \cdot X} X^{\nu} e^{i\left(1-\tau_{2}\right) k \cdot X}\right) \\
& +\int_{0}^{1} d \tau_{1} e^{-2 \pi i \tau_{1}} \int_{\tau_{1}}^{1} d \tau_{2} e^{2 \pi i \tau_{2}} \operatorname{Tr}\left(e^{i \tau_{1} k \cdot X} X^{\mu} e^{i\left(\tau_{2}-\tau_{1}\right) k \cdot X} X^{\nu} e^{i\left(1-\tau_{2}\right) k \cdot X}\right) \\
& +\cdots
\end{aligned}
$$

We see that in all the cases above, the matrix model part of the result has the generic form given in (4.8). It should also be noted that for the vertex operator $V_{3}$, the result of the calculation gives a linear sum of matrix model expressions of the type in (4.8). The above calculations can be easily generalized to higher levels and it turns out that they have the same generic features.

Before we end this section, let us come back to the conserved operators $O_{\mu \nu}^{(n)}(k)$ identified in the previous section. From the above calculations, we see that the operator for $n=2$ is dual to the closed string mode given by the vertex operator $V_{3}$, and so on. Conservation of these may, therefore, seem puzzling since $k_{\mu} V_{3}^{\mu \nu}$ is not a total worldsheet derivative. However, the difference from a total derivative is essentially given by $k_{\mu} k_{\nu} V_{2}^{\mu \nu \lambda}$. The matrix model operator dual to this operator vanishes, as can easily be seen from the above calculations. It is these type of unexpected identities satisfied by the matrix model operators that are responsible for the infinite set of conserved operators that we have identified here.

\section{Symmetries of the generating functional of Wilson line correlators}

In the previous sections we have established that the correlation functions of the gauge theory operators that appear in the harmonic expansion of a generic Wilson line $W_{C}$ are invariant under reparametrizations, even though the modes $\alpha_{n}$ of the periodic function 
$w(\sigma)$ change in a complicated way. In this section we will study a non-trivial consequence of this fact.

Let us add to the non-commutative gauge theory action source terms for all the operators that appear in the harmonic expansion:

$$
\int d^{D} k \sum_{n_{1}, n_{2}, \cdots=1}^{\infty} \sum_{m_{1}, m_{2}, \cdots=1}^{\infty} \Phi_{\mu_{1}, \mu_{2}, \ldots ; \nu_{1}, \nu_{2}, \ldots}^{n_{1}, n_{2}, \ldots ; m_{1}, m_{2}, \ldots}(k) O_{\mu_{1}, \mu_{2}, \ldots ; \nu_{1}, \nu_{2}, \ldots}^{n_{1}, n_{2}, \ldots ; m_{1}, m_{2}, \ldots}(k)
$$

A more elegant way of writing this is the following. Let us introduce the "closed string field" $\Phi_{k}[w] \equiv \Phi_{k}\left(\alpha_{1}, \alpha_{2}, \cdots\right)$ 凹 and define the sources as its "moments":

$$
\begin{aligned}
& \Phi_{\mu_{1}, \mu_{2}, \ldots ; \nu_{1}, \nu_{2}, \ldots}^{n_{1}, n_{2}, \ldots ; m_{1}, m_{2}, \ldots}(k) \\
& =\int \prod_{n=1}^{\infty} d \alpha_{n} d \alpha_{n}^{*} \alpha_{n_{1} \mu_{1}} \alpha_{n_{2} \mu_{2}} \cdots \alpha_{m_{1} \nu_{1}}^{*} \alpha_{m_{2} \nu_{2}}^{*} \cdots \Phi_{k}\left(\alpha_{1}, \alpha_{2}, \cdots\right) .
\end{aligned}
$$

We may then rewrite (6.1) in the compact form

$$
\int d^{D} k \int \prod_{n=1}^{\infty} d \alpha_{n} d \alpha_{n}^{*} \Phi_{k}\left(\alpha_{1}, \alpha_{2}, \cdots\right) W_{C_{0}}\left(\alpha_{1}, \alpha_{2}, \cdots\right)
$$

where we have explicitly indicated the parametrization of the contour $C$ in terms of the straight line $C_{0}$ (which is given by the starting point $y_{0}(0)$ and the the momentum $k$ ) and the modes of the periodic function $w$.

In the above we have assumed that the Wilson line is expanded in terms of the modes of the periodic function $w$. We could equally well have used the parametrization of the contour $C$ in terms of $C_{0}^{\prime}$ and $w^{\prime}$ and then the Wilson line would be expanded in terms of the modes of $w^{\prime}$. Replacing $W_{C_{0}}\left(\alpha_{1}, \alpha_{2}, \cdots\right)$ by $W_{C_{0}^{\prime}}\left(\alpha_{1}^{\prime}, \alpha_{2}^{\prime}, \cdots\right)$ in (6.3) has the effect of changing the sources because the primed modes are non-trivial functions of the unprimed ones and because the function $\Phi_{k}\left(\alpha_{1}, \alpha_{2}, \cdots\right)$ has not been changed. These transformed sources, $\Phi^{\prime}$, are now coupled to the primed operators $O^{\prime}$ discussed in the previous section. However, since the correlation functions of $O^{\prime}$ are identical to those of the operators $O$, it follows that the generating functional of the correlators of these operators, which is given by

$$
Z[\Phi]=<\exp \left\{\int d^{D} k \int \prod_{n=1}^{\infty} d \alpha_{n} d \alpha_{n}^{*} \Phi_{k}\left(\alpha_{1}, \alpha_{2}, \cdots\right) W_{C_{0}}\left(\alpha_{1}, \alpha_{2}, \cdots\right)\right\}>
$$

remains unchanged, i.e.

$$
Z\left[\Phi^{\prime}\right]=Z[\Phi]
$$

How are the transformed sources $\Phi^{\prime}$ related to $\Phi$ ? To see this it is useful to look at some explicit examples. The first non-trivial example is provided by the sources which

\footnotetext{
${ }^{11}$ The condition $\Phi_{-k}^{*}=\Phi_{k}$ is needed to ensure that the source terms in the action are real
} 
couple to gauge theory operators that are dual to the massless closed string modes. In terms of the definition in (6.2) these sources are given by

$$
\Phi_{\mu ; \nu}^{1 ; 1}(k)=\int \prod_{n=1}^{\infty} d \alpha_{n} d \alpha_{n}^{*} \alpha_{1 \mu} \alpha_{1 \nu}^{*} \Phi_{k}\left(\alpha_{1}, \alpha_{2}, \cdots\right)
$$

After a reparametrization of the contour $C$ these sources transform to

$$
\Phi_{\mu ; \nu}^{1 ; 1}(k)^{\prime}=\int \prod_{n=1}^{\infty} d \alpha_{n} d \alpha_{n}^{*} \alpha_{1 \mu}^{\prime} \alpha_{1 \nu}^{\prime *} \Phi_{k}\left(\alpha_{1}, \alpha_{2}, \cdots\right)
$$

Using (3.8) in this and retaining terms only upto first order in $\epsilon$, we get

$$
\begin{aligned}
\Phi_{\mu ; \nu}^{1 ; 1}(k)^{\prime}-\Phi_{\mu ; \nu}^{1 ; 1}(k)= & -k_{\mu} \epsilon_{1} \Phi_{; \nu}^{; 1}(k)-k_{\nu} \epsilon_{1}^{*} \Phi_{; \mu}^{; 1}(-k)+\left\{4 \pi i \epsilon_{1}^{*} \Phi_{\mu ; \nu}^{2 ; 1}(k)\right. \\
& \left.-2 \pi i \epsilon_{2} \Phi_{; \mu, \nu}^{; 1,1}(k)+6 \pi i \epsilon_{2}^{*} \Phi_{\mu ; \nu}^{3 ; 1}(k)+\text { c.c. }\right\}+\cdots
\end{aligned}
$$

where,

$$
\Phi_{; \mu}^{; 1}(k)=\int \prod_{n=1}^{\infty} d \alpha_{n} d \alpha_{n}^{*} \alpha_{1 \mu}^{*} \Phi_{k}\left(\alpha_{1}, \alpha_{2}, \cdots\right)
$$

follows from the definition (6.2) and similarly for $\Phi_{; \mu, \nu}^{; 1,1}(k)$ etc. The 'c.c.' in (6.8) stands for complex conjugation together with $\mu \leftrightarrow \nu$ and $k \rightarrow-k$ and the dots represent terms involving $\epsilon_{3}$ and higher modes of $\epsilon(\sigma)$.

Let us first focus on terms proportional to $\epsilon_{1}$ on the right hand side of (6.8). We see that the symmetric part of the source $\Phi_{\mu ; \nu}^{1 ; 1}(k)$, which couples to the gauage theory operator dual to the closed string graviton, transforms just like the linearised transformation of metric under general coordinate transformations. Similarly, the antisymmetric part of $\Phi_{\mu ; \nu}^{1 ; 1}(k)$ transforms just like the antisymmetric tensor field under gauge transformations. The presence of these space-time symmetries implies that the longitudinal modes of the operators dual to graviton and antisymmetric tensor field decouple. In string theory, this corresponds to the decoupling of the spurious states

$$
L_{-1} \tilde{a}_{1 \mu}^{\dagger}\left|0 ; k>, \quad \tilde{L}_{-1} a_{1 \mu}^{\dagger}\right| 0 ; k>,
$$

where $a_{n}, \tilde{a}_{n}$ are the left and right moving oscillator modes of the closed string and the $L_{n}, \tilde{L}_{n}$ are the corresponding virasoro generators. From the transformation of $\Phi_{\mu ; \nu}^{1 ; 1}(k)$ we see that the non-commutative gauge theory/matrix model analogues of these states are the fields $\Phi_{; \mu}^{; 1}(k)$ and $\Phi_{\mu ;}^{1 ;}(k)$. Using

At level two we have several sources, $\Phi_{\mu, \nu ; \lambda \rho}^{1,1 ; 1,1}(k), \Phi_{\mu, \nu ; \lambda}^{1,1 ; 2}(k), \Phi_{\mu ; \nu}^{2 ; 2}(k)$ and their congugates.

$$
\alpha_{2}^{\prime}=\alpha_{2}-k \epsilon_{2}+4 \pi i \epsilon_{0} \alpha_{2}+2 \pi i \epsilon_{1} \alpha_{1}+2 \pi i \sum_{n=1}^{\infty}\left((n+2) \epsilon_{n}^{*} \alpha_{n+2}-n \epsilon_{n+2} \alpha_{n}^{*}\right),
$$


we find that the source $\Phi_{\mu ; \nu}^{2 ; 2}(k)$ transforms as

$$
\begin{aligned}
\Phi_{\mu ; \nu}^{2 ; 2}(k)^{\prime}-\Phi_{\mu ; \nu}^{2 ; 2}(k)= & -k_{\mu} \epsilon_{2} \Phi_{; \nu}^{; 2}(k)-k_{\nu} \epsilon_{2}^{*} \Phi_{; \mu}^{; 2}(-k)+\left\{2 \pi i \epsilon_{1} \Phi_{\mu ; \nu}^{1 ; 2}(k)\right. \\
& \left.+6 \pi i \epsilon_{1}^{*} \Phi_{\mu ; \nu}^{3 ; 2}(k)+8 \pi i \epsilon_{2}^{*} \Phi_{\mu ; \nu}^{4 ; 2}(k)+\text { c.c. }\right\}+\cdots
\end{aligned}
$$

Similarly,

$$
\begin{aligned}
\Phi_{\mu, \nu ; \lambda}^{1,1 ; 2}(k)^{\prime}-\Phi_{\mu, \nu ; \lambda}^{1,1 ; 2}(k)= & \left(-k_{\mu} \epsilon_{1} \Phi_{\nu ; \lambda}^{1 ; 2}(k)+4 \pi i \epsilon_{1}^{*} \Phi_{\mu, \nu ; \lambda}^{2,1 ; 2}(k)-2 \pi i \epsilon_{2} \Phi_{\nu ; \mu, \lambda}^{1 ; 1,2}(k)+6 \pi i \epsilon_{2}^{*} \Phi_{\mu, \nu ; \lambda}^{3,1 ; 2}(k)\right) \\
& +(\mu \leftrightarrow \nu)-k_{\lambda} \epsilon_{2}^{*} \Phi_{\mu, \nu ;}^{1,1 ;}(k)-6 \pi i \epsilon_{1} \Phi_{\mu, \nu ; \lambda}^{1,1 ; 3}(k)-8 \pi i \epsilon_{2} \Phi_{\mu, \nu ; \lambda}^{1,1 ; 4}(k) \\
& -2 \pi i \epsilon_{1}^{*} \Phi_{\mu, \nu ; \lambda}^{1,1 ; 1}(k)+\cdots
\end{aligned}
$$

One can also similarly work out the transformation of the source $\Phi_{\mu, \nu ; \lambda \rho}^{1,1 ; 1,1}(k)$. It is easy to see that in all the cases, the gauge transformation due to $\epsilon_{1}$ at this level is consistent with the decoupling of operators whose string theory analogues are the spurious states

$$
L_{-1} a_{1 \mu}^{\dagger} \tilde{a}_{2 \nu}^{\dagger}\left|0 ; k>, \quad L_{-1} a_{1 \mu}^{\dagger} \tilde{a}_{1 \nu}^{\dagger} \tilde{a}_{1 \lambda}^{\dagger}\right| 0 ; k>, \quad \tilde{L}_{-1} a_{2 \mu}^{\dagger} \tilde{a}_{1 \nu}^{\dagger}\left|0 ; k>, \quad \tilde{L}_{-1} a_{1 \mu}^{\dagger} a_{1 \nu}^{\dagger} \tilde{a}_{1 \lambda}^{\dagger}\right| 0 ; k>,
$$

etc. We should note here that the first term in the curly brackets on the right hand side of (6.8) is also of this type. This is because, as we observed in the computation of the disc amplitude involving the vertex operator $V_{3}$ in the last section, a closed string vertex operator at a given level is in general dual to a linear combination of gauge theory operators upto that level एँ.

We can also now interpret the gauge transformation due to $\epsilon_{2}$ in terms of string theory. From the above transformations, it is clear that this corresponds to the spurious states

$$
L_{-2} \tilde{a}_{2 \mu}^{\dagger}\left|0 ; k>, \quad \tilde{L}_{-2} a_{2 \mu}^{\dagger}\right| 0 ; k>
$$

etc.

At higher levels and for higher modes of $\epsilon(\sigma)$ a similar pattern is confirmed and one finds that the gauge transformations of the sources are in one-to-one correspondence with the spurious states of string theory. In string theory this gauge symmetry allows one to remove the entire tower of oscillator modes corresponding to a single coordinate degree of freedom. The analogue of this here is the fact that in the matrix model one can always diagonalize one of the matrices by a unitary transformation, so the actual dynamical degrees of freedom contain one less matrix. This fact is not so obvious in terms of the operators that appear in the harmonic expansion of the Wilson line, since these operators are manifestly invariant under unitary transformations of the matrices. However, in the space-time interpretation of these operators, in which each matrix is interpreted as a coordinate, this fact reappears as a gauge symmetry. In this sense it is natural and consistent to find that the gauge symmetry of the generating functional $Z[\Phi]$ is identical to the gauge symmetry of closed string theory.

\footnotetext{
${ }^{12}$ Here, by the level of a gauge theory operator we mean the sum of positive (or negative) numbers in the mode exponentials. For example, the level of the operator $O_{\mu \nu}^{(n)}$ given in 4.13 is $n$.
} 


\section{Discussion}

In this paper we have investigated the proposal that the harmonic expansion of an open Wilson line, defined on a generic contour, around a certain straight line contour, contains gauge theory operators dual to all the modes of the closed string. This was motivated by the observation that the first two operators in the expansion are dual to the tachyon and the massless modes of the closed string. We argued that the gauge theory operators that appear in the harmonic expansion satisfy an analogue of the level matching condition. Further evidence for our proposal came from some explicit computations of disc amplitudes. We also discussed space-time gauge symmetries of the generating functional of correlation functions of these operators. The gauge symmetries are identical to what one finds in the field theory of closed strings. We showed that the symmetries are a direct consequence of the reparametrization invariance of the original Wilson line.

An aspect of the present investigations that needs to be clarified is the absence of any condition on the dimensions of the target space. Presumably this is related to the fact that in the bosonic string there is no condition on the background fields in the sigmamodel at the classical level. Our analysis here has also been classical. It is conceivable that the formal reparametrization invariance of the Wilson line is not realized in the generating functional defined in (6.4) at the quantum level unless the sources satisfy certain conditions, including a condition on the number of space-time dimensions. In the supersymmetric case, ensuring space-time supersymmetry, for example by ensuring kappa-symmetry in the Green-Schwarz formalism, leads to a restriction on the background fields already at the classical level. It would, therefore, be very intersting to generalize the present analysis to the case of the superstring.

Our analysis here has been essentially kinematical, relying only on the symmetries of the system. Investigating its dynamics is clearly necessary for further progress. For example, what role do Schwinger-Dyson or Loop equations play in the story? These equations represents dynamical constraints on the correlation functions of the gauge theory operators. It would be interesting to find out what kind of constraints they imply on the functional form of the generating functional $Z[\Phi]$.

Another issue related to the quantum dynamics of the present system is the following. In the representation theory of Virasoro algebra, the critical dimension is associated with further reducibility due to the emergence of null states. After eliminating the null states, we are left with the light-cone gauge like physical degrees of freedom. We need to identify the central charge associated to the quantum Virasoro algebra for the Wilson lines to determine the critical dimension of the closed string theory dual to it. Quantum dynamics of Wilson lines certainly plays an important role here and in principle it can be extracted from the Loop equations.

Finally, we point out that we may also regard (6.4) as the partition function of bosonic version of the matrix model in weak background fields 피. Actually since all possible terms

\footnotetext{
${ }^{13}$ Various aspects of matrix models in background fields have been studied in [22, 23, 24, 25, 26, 27,
} 
in the matrix theory action can be obtained from special sources [4], the entire action can be thought of as just matrix theory operators coupled to sources. This is very similar to the world-sheet sigma model action for the bosonic string in background fields. It would be interesting to see how far this analogy holds. In particular, does quantum consistency of the matrix model impose restriction on the sources? One particular consistency condition that we should require of the quantum theory is the reparametrization invariance or equivalently the gauge invariance of (6.4). It would be very interesting to see whether this condition is enough to recover the correct string dynamics within this framework.

\section{References}

[1] S.R. Das and Soo-Jong Rey, "Open Wilson lines in non-commutative gauge theory and tomography of holographic dual supergravity", Nucl.Phys. B590 (2000) 453, hep-th/0008042.

[2] D.J. Gross, A. Hashimoto and N. Itzhaki, "Observables of non-commutative gauge theories", hep-th/0008075.

[3] H. Liu, “*_Trek II”, hep-th/0011125.

[4] S.R. Das and S. Trivedi, "Supergravity couplings to noncommutative branes, open Wilson line and generalized star products", JHEP 0102 (2001) 046, hep-th/0011131.

[5] Y. Okawa and H. Ooguri, "How noncommutative gauge theories couple to gravity", Nucl.Phys. B599 (2001) 55, hep-th/0012218; "Energy momentum tensors in matrix theory and in noncommutative gauge theories", hep-the/0103124.

[6] H. Liu and J. Michelson, "Supergravity couplings of noncommutative D-branes", hep-th/0101016.

[7] N. Ishibashi, S. Iso, H. Kawai and Y. Kitazawa, "Wilson loops in non-commutative Yang-Mills", Nucl. Phys. B573 (2000) 573, hep-th/9910004.

[8] A. Dhar and Y. Kitazawa, "Loop Equation and Wilson line Correlators in Noncommutative Gauge Theories", hep-th/0104021.

[9] O. Andreev and H. Dorn, "On open string sigma-model and non-commutative gauge fields", Phys. Lett. B476 (2000) 402, hep-th/9912070.

[10] Soo-Jong Rey and R. von Unge, "S-duality, non-critical open string and noncommutative gauge theory", Phys.Lett. B499 (2001) 215, hep-th/0007089.

$28,29,30,31$

${ }^{4}$ For example, the commutator squared term in the matrix model action corresponds to the source $\Phi_{\mu ; \nu}^{1 ; 1}(k) \sim \partial_{k_{\mu}} \partial_{k_{\nu}} \delta^{D}(k)$. This follows from the identity $\left.\operatorname{Tr}\left[X_{\mu}, X_{\nu}\right]^{2} \sim \partial_{k_{\mu}} \partial_{k_{\nu}} O_{\mu ; \nu}^{1 ; 1}(k)\right|_{k=0}$. 
[11] A. Dhar and S.R. Wadia, "A note on gauge invariant operators in non-commutative gauge theories and the matrix model", Phys. Lett. B495 (2000) 413, hepth/0008144.

[12] M. Rozali and M. van Raamsdonk, "Gauge-Invariant Correlators in Noncommutative Gauge Theory", hep-th/0012065.

[13] A. Dhar and Y. Kitazawa, "High energy behaviour of Wilson lines", JHEP 0102 (2001) 004, hep-th/0012170.

[14] D. Berenstein and R.G. Leigh, "Observations on non-commutative field theories in coordinate space", hep-th/0102158.

[15] N. Ishibashi, H. Kawai, Y. Kitazawa and A. Tsuchiya, "A Large-N Reduced Model as Superstring", Nucl. Phys B498 (1997) 467, hep-th/9612115.

[16] I. Bars, Phys. Lett. 245B (1990) 35.

[17] D.B. Fairlie, P. Fletcher and C.Z. Zachos, J. Math. Phys. 31 (1990) 1088.

[18] S. Rey, "Heterotic M(atrix) Strings and Their Interactions", Nucl. Phys. B502 (1997) 170, hep-th/9704158.

[19] G.T. Horowitz and L. Susskind, "Bosonic M Theory", hep-th/0012037.

[20] K. Okuyama, "Comments on open Wilson lines and generalized star products", Phys.Lett. B506 (2001) 377, hep-th/0101177.

[21] N. Ishibashi, "A relation between commutative and non-commutative descriptions of D-branes", hep-th/9909176.

[22] M.R. Douglas, "D-branes in curved space", Adv. Theor. Math. Phys. 1 (1998) 198, hep-th/9703056 and "D-branes and matrix theory in curved space", Nucl. Phys. Proc. Suppl. 68 (1998) 381, hep-th/9707228.

[23] M.R. Douglas, A. Kato and H. Ooguri, "D-brane actions on Kaehler manifolds", Adv. Theor. math. Phys. 1 (1998) 237, hep-th/9708012.

[24] M.R. Douglas and H. Ooguri, "Why matrix theory is hard", Phys. Lett. B425 (1998) 71, hep-th/9710178.

[25] D. Kabat and W.I. Taylor, "Linearized supergravity from matrix theory", Phys. Lett. B426 (1998) 297, hep-th/9712185.

[26] W.I. Taylor and M. van Raamsdonk, "Supergravity currents and linearized interactions for matrix theory configurations with fermionic backgrounds", JHEP 9904 (1999) 013, hep-th/9812239.

[27] Y. Kimura and Y. Kitazawa, "Supercurrent Interactions in Noncommutative YangMills and IIB Matrix Model", Nucl. Phys. 598B (2001) 73, hep-th/0011038. 
[28] W.I. Taylor and M. van Raamsdonk, "Multiple D0-branes in weakly curved backgrounds", Nucl. Phys. B558 (1999) 63, hep-th/9904095.

[29] W.I. Taylor and M. van Raamsdonk, "Multiple Dp-branes in weak background fields", Nucl. Phys. B573 (2000) 703, hep-th/9910052.

[30] R.C. Myers, "Dielectric-branes", JHEP 9912 (1999) 022, hep-th/9910053.

[31] A. Dasgupta, H. Nicolai and J. Plefka, "vertex operators for the supermembrane", JHEP 0005 (2000) 007, hep-th/0003280. 